\title{
Management of patients with type 2 diabetes mellitus in the Grand-Aides Program
}

\author{
Rebekah M. Compton*1, Kimberly S. Bednar ${ }^{1}$, Peggie E. Donowitz ${ }^{2}$, M. Norman Oliver ${ }^{3}$ \\ ${ }^{1}$ Department of Family Medicine, University of Virginia, USA \\ ${ }^{2}$ Grand-Aides USA, Houston, Texas, USA \\ ${ }^{3}$ Commonwealth of Virginia, Commissioner of Health, USA
}

Received: September 13, 2019

Accepted: October 30, 2019

Online Published: November 17, 2019

DOI: $10.5430 /$ jnep.v10n3p1

URL: https://doi.org/10.5430/jnep.v10n3p1

\begin{abstract}
Objective: To evaluate the Grand-Aides Program for patients with type 2 diabetes mellitus (T2DM) according to the variables of body weight, blood pressure, medication adherence, and hospital consultation and readmissions.

Methods: Patients ages 18 years or older with a past medical history of T2DM, hypertension (HTN), and/or obesity and who were recently seen in the emergency department (ED) or recently admitted to the hospital were eligible to enroll in the Grand-Aides Program. Eligible patients were identified after hospital or ED discharge and were asked to enroll in the in-home based program from March 2016 through June 2018. In-home visit protocol was defined prior to patient enrollment with intense in-home visits during the first weeks of enrollment followed by monthly visits for the duration of enrollment in the program. In-home visit frequency was adjusted on as needed basis so that patients at higher risk for ED visits or hospitalization were seen more frequently. In-home visits were performed by trained Grand-Aide who for the purpose of this study was a certified nursing assistant (CNA). The Grand-Aide underwent eighty hours of didactic training which included visit protocols, visit schedules, and data collection. The one-on-one in-home patient with every visit were supervised by a registered nurse (RN) or nurse practitioner (NP) via video or telephone contact near the conclusion of the visit. Active patients at the University of Virginia Family Medicine clinic were eligible for enrollment. Fifty-seven patients with T2DM worked with Grand-Aides for three months and an additional forty-eight T2DM patients worked with Grand-Aides for twelve months. Emergency department visits, all 30-day hospital readmissions, as well as blood pressure readings, medication adherence, weights, and glycated hemoglobin (HbAlc) were compared with the prior twelve months.

Results: Systolic $(p<.001)$ and diastolic $(p<.01)$ blood pressures decreased $(p<.01)$ at 1 year. At baseline 56 percent of the patients had a systolic blood pressure of $>130 \mathrm{mmHg}$ despite treatment; after 12 months, 48 percent of these were $<130$. In those whose baseline diastolic blood pressure was $>90 \mathrm{mmHg}, 100$ percent had diastolic blood pressure $<90 \mathrm{mmHg}$ at 1 year. Medication adherence by ARMS test at 1 year was 94 percent. Despite trending downward, weight and HbA1c did not change significantly. In the preceding, 58 percent had at least one ED visit, which was reduced by 50 percent $(p<.01)$ with Grand-Aides; 30-day all-cause readmissions reduced by 50 percent to 6.3 percent.

Conclusions: The Grand-Aides program was associated with a significant change in blood pressure control, high medication adherence and reductions in ED visits and readmissions that compare favorably with published comparative data. For systems "at risk" for preventable increased health care expense burden, the Grand-Aides program can result in significant savings.
\end{abstract}

Key Words: Grand-Aides, Cost reduction care management, Home care, Type 2 diabetes mellitus, Diabetes care management, Hypertension, Blood pressure management

*Correspondence: Rebekah M. Compton; Email: rc8b@ Virginia.edu; Address: 1215 Lee St., P.O. Box 800729, Charlottesville, VA 22908, USA. 


\section{INTRODUCTION}

Management of patients with Type 2 Diabetes Mellitus (T2DM) is notoriously challenging, due to the patient's nonadherence to prescribed diets and medication regimens. When in many cases, lack of adherence contributes to worsening or uncontrolled diabetes. Numerous attempts to improve diabetes management have been made including various published diet regimes, medication adherence aids, and mobile apps. ${ }^{[1-3]}$ Hospital readmission in patients with T2DM is over 26 percent, making T2DM a major risk factor for readmission. ${ }^{[4]}$ The most successful management, and one that is endorsed by the American Diabetes Association in their Standards of Medical Care, is the use of lay "coaching". [5]

Recently, a program called "Grand-Aides", which employed lay coaches, reported a 92 percent medication adherence at 30 days after hospital discharge in Medicare patients with heart failure. ${ }^{[6]}$ This report notes an 82 percent reduction in all-cause 30-day readmissions; at 6 months, all-cause readmissions were reduced 71 percent and ED visits decreased 77 percent.

\section{Grand-Aides}

A Grand-Aide is a nurse aide who makes frequent home visits after hospital discharge with every visit supervised by a RN or NP. Close to the end of the visit, the Grand-Aide contacts the NP supervisor either by video chat or by phone which allows the supervisor to interact with the patient and family.

These live interactions build patient provider relationships and trust by providing in the moment health promotion recommendations. It is important to note, the Grand-Aide make no independent clinical decisions for the patient, but instead carry out the RN/NP's plan of care. When a RN is utilized as the supervisor, then RN will interact with the patient's physician or Advanced practice provider (APP) to receive input and assist with care management decisions.

In-home visits are a pivotal component of this program. This allows the Grand-Aide and supervisor to achieve a more comprehensive view of how the patient lives and how they manage their health at home. Initially, in-home visits are scheduled for two to three times weekly for the first weeks of enrollment. However, the visits decrease over time depending upon the complexity of the patient's needs. Continued patient contact with a telemedicine platform or more frequent in-home visits is determined by NP supervisor or the patient's care team. ${ }^{[6-8]}$

The Grand-Aides program differs from certified home health agencies in the following. Grand-Aides: 1) Have every nurse aide visit supervised in real-time by an $\mathrm{RN}$ or NP which includes observing the patient and answering questions; 2) Spending as much time as necessary with the patient on each visit; 3) Providing in-home visits for high-risk patients for as long as a need is present (e.g. $>60$ days); 4) Addressing the patient's medication adherence and health literacy regarding risk factors for increased health complications; 5) Carrying for patients with relatively straightforward to extremely complex conditions.

In the present research, we used the same procedures as for hospital discharge for patients with T2DM, offering them Grand-Aides in the outpatient clinic.

\section{METHODS}

\subsection{Patients}

Beginning March 1, 2016 all patients with a diagnosis of T2DM in the Family Medicine Clinic at the University of Virginia Medical Center were presented with the opportunity to participate in the Grand-Aides program. Patient could be enrolled in the program within one week of hospital discharge. The last patient was enrolled on April 13, 2018. Various approaches were used to enroll patients. A report within the electronic medical record allowed for identification of patients. Either the patient received a cold call or the primary care practitioner (PCP) was notified of the patient's eligibility for the program. When the patient's PCP (physician or Nurse Practitioner) offered the program, the Grand-Aides NP supervisor followed-up immediately at time of the clinic visit or contacted the patient over the phone to schedule initial in-home visit. Follow-up for 84 percent was for one year; data were included for the other thirteen patients with at least three months of follow-up. There was no control arm, so patients were their own control for pre- and post-study comparison.

The research was approved by the University of Virginia Institutional Review Board.

\subsection{Grand-Aides procedures}

While the more minute details of the procedures are described elsewhere, ${ }^{[6-8]}$ a brief overview of the process is described here. The procedure modified for the clinic was that patients received home visits two times the first four week after the clinic visit, then once weekly for the following four weeks, with once monthly visits thereafter. Increased in-home visits or follow up telephone calls were scheduled based on patient's needs at the discretion of the NP supervisor or the primary practitioner. Patient's and family members were also at liberty to contact the Grand-Aides to request nonscheduled in-home visits.

On the first in-home visit, a "medication reconciliation" was 
performed where the Grand-Aide would ask the patient to gather all medications in the home. The Grand Aide and the patient would review each medication bottle and have the patient described how they are taking their medications. The Grand-Aide would then contact the NP supervisor via video chat or phone. The NP supervisor and Grand-Aide compared the current medication list on file with the medications actually taken. Following the call, the medication variations and description of the visit were documented and shared with the provider. The patient's provider was then able to more accurately address medications at subsequent clinic visits. During the initial home visit, the Grand-Aide administered a specifically-tailored symptom questionnaire (to include questions related to patient specific diagnosis, e.g. chronic obstructive pulmonary disease). Medication adherence was assessed using the using the criteria of the Adherence to Refills and Medications Scale (ARMS) test and by observation assessed by the GAs and supervisor ${ }^{[9]}$ to "All of the time," "Most of the time," "Some of the time," and "None of the time." Substantial adherence was considered answering at least "Most of the time." It is important to note that patients at baseline were asked only to complete the ARMS questionnaire. Therefore, baseline responses could not be verified by the Grand-Aides as they had not observed the adherence and the baseline number could be lower.

Medication adherence has been a long standing concerning in health management. Hippocrates believed that patients "faked ingestion of their medications". ${ }^{[10]}$ This problem remains to the present and therefore, in the Grand-Aides program, after the baseline, the patient answers to the ARMS test are verified by Grand-Aides observation.

\subsection{Data and analysis}

Demographics were recorded on admission to the program. At baseline, 3, 6 and 12 months, the following were recorded: weight, blood pressure, HbA1c, and medication adherence. For the patients seen for 1 year, all visits to the Emergency Department (ED), excluding trauma were recorded. All admissions to the hospital (excluding trauma as the primary diagnosis) were recorded. Those admissions that occurred within 30 days of a previous admission were counted as "all cause 30-day readmissions."

Given that all patients were under active management on admission to the Grand-Aides program, those patients with "abnormal values" were analyzed separately from those with "normal values" at the onset. "Abnormal" was considered as $>199$ pounds for weight, either >130 mmHg. systolic blood pressure or $>90 \mathrm{mmHg}$. diastolic blood pressure (or both), and $>7.0 \mathrm{mmol} / \mathrm{mol}$ for Hemoglobin A1c. The reasoning behind that was that management could not be considered

Published by Sciedu Press successful, for example, if a patient weighing $<200$ pounds after 1 year weighed $<200$ pounds initially. For Emergency Dept visits and readmissions, the time period before admission to the program was taken as the control for the time period after beginning the program, e.g. for readmissions within 6 months after the program, the readmissions within 6 months before the program for each patient.

Each patient served as their own control with change from baseline. Paired $t$-tests were used and statistical significance was considered at $p<.05$.

\section{RESULTS}

Patients aged 18 years old or older were eligible to enroll in the Grand Aides Program. The medical records of 57 patients were analyzed; 57 had 3 months of follow-up, 53 had 6 months and 48 were followed for 1 year ( 1 death occurred between 6 and 12 months). The age was 58 (mean +/- SD) and Median was 59; 22 were male, 63 female; 50 non-Caucasian (46 African American, 2 Hispanic) and 35 Caucasian.

\subsection{Medical outcomes}

The outcomes data are shown in Table 1. At baseline, 60 percent of the patients had a weight of 200 pounds or more, 56 percent with a systolic blood pressure greater than 130 $\mathrm{mmHg}$., 19 percent with a diastolic blood pressure greater than $90 \mathrm{mmHg}$. and 60 percent had a hemoglobin A1c greater than 8.0 percent $[64 \mathrm{mmol} / \mathrm{mol}] .^{[17]}$ The results for these patients are also shown in rows 2-5 of Table 1.

Those with an abnormal weight had an average weight of 274 pounds. Despite a mean reduction of weight to 250 pounds at 3 months and 255 pounds at 12 months, the standard deviations were large, and the differences were not statistically significant. The nurse aides noted with certain patients that food scarcity and dependence on food banks for food may have contributed to lack of weight loss as well. The impact of food scarcity would need to be studied in a subsequent study. The weights in this group of those $>199$ pounds ranged from 200 to 511 pounds. These patients showed a sustained significant decrease in systolic blood pressure.

The patients that began with an abnormal systolic blood pressure averaging $146 \mathrm{~mm} \mathrm{Hg}$., showed early significant reductions beginning at 3 months and maintained at 1 year at a mean of $133 \mathrm{mmHg}(p<.0001)$. Systolic blood pressure in this group was significantly reduced at 3 months from $155 \mathrm{mmHg}$ to $140 \mathrm{mmHg}(p<.01), 6$ months at $137 \mathrm{mmHg}$ $(p<.01)$ and then to $139 \mathrm{mmHg}$ at 1 year $(p=.06)$. At baseline 56 percent of patients had abnormal systolic blood pressure despite treatment; after 12 months, 48 percent of 
these came under control. These patients also had a significant decrease in diastolic blood pressure from $86 \mathrm{mmHg}$ to $76 \mathrm{mmHg}$ which remained a stable improvement at one year $(p<.01)$. No patient had diastolic hypertension without systolic hypertension. In the 19 percent of patients whose diastolic blood pressure was $>90 \mathrm{mmHg}$ at baseline, they maintained a significant decrease in diastolic blood pressure at 1 year with 100 percent having controlled diastolic blood pressure.

The HbA1c fell early at 3 months from 9.6 percent [ 81 $\mathrm{mmol} / \mathrm{mol}$ (to 9.0 percent $[75 \mathrm{mmol} / \mathrm{mol}](p<.025)$. The mean continued lower at 9.3 percent $[78 \mathrm{mmol} / \mathrm{mol}]$ at 6 and 12 months but this was not statistically different from the baseline 9.6 percent $[81 \mathrm{mmol} / \mathrm{mol}]$.

Table 1. Medical outcomes for patients with type 2 diabetes and Grand-Aides

\begin{tabular}{|c|c|c|c|c|c|c|c|c|c|c|c|c|c|c|c|c|}
\hline & \multicolumn{4}{|c|}{ BASELINE $\mathbf{n}=57$} & \multicolumn{4}{|c|}{3 MONTHS n = 57} & \multicolumn{4}{|c|}{6 MONTHS n = 53} & \multicolumn{4}{|c|}{12 MONTHS n = 48} \\
\hline & $\begin{array}{c}\text { WEIGHT } \\
\text { Pounds }\end{array}$ & $\begin{array}{c}\text { SBP } \\
\text { mmHg. }\end{array}$ & $\begin{array}{c}\text { DBP } \\
\text { mmHg. }\end{array}$ & $\begin{array}{c}\text { A1c } \\
\text { Percent }\end{array}$ & $\begin{array}{l}\text { WEIGHT } \\
\text { Pounds }\end{array}$ & $\begin{array}{c}\text { SBP } \\
\text { mmHg. }\end{array}$ & $\begin{array}{c}\text { DBP } \\
\text { mmHg. }\end{array}$ & $\begin{array}{c}\text { A1c } \\
\text { Percent }\end{array}$ & $\begin{array}{c}\text { WEIGHT } \\
\text { Pounds }\end{array}$ & $\begin{array}{c}\text { SBP } \\
\text { mmHg. }\end{array}$ & $\begin{array}{c}\text { DBP } \\
\text { mmHg. }\end{array}$ & $\begin{array}{c}\text { A1c } \\
\text { Percent }\end{array}$ & $\begin{array}{c}\text { WEIGHT } \\
\text { Pounds }\end{array}$ & $\begin{array}{c}\text { SBP } \\
\text { mmHg. }\end{array}$ & $\begin{array}{c}\text { DBP } \\
\text { mmHg. }\end{array}$ & $\begin{array}{c}\text { A1c } \\
\text { Percent }\end{array}$ \\
\hline All patients & $230 \pm 75$ & $134 \pm 18$ & $79 \pm 13$ & $8.3 \pm 2.3$ & $211 \pm 50$ & $130 \pm 13$ & $78 \pm 10$ & $8.3 \pm 2.0$ & $217 \pm 54$ & $131 \pm 15$ & $77 \pm 12$ & $8.0 \pm 2.2$ & $217 \pm 54$ & $129 \pm 16$ & $75 \pm 11$ & $8.2 \pm 2.0$ \\
\hline $\begin{array}{c}\text { WEIGHT } \\
>199\end{array}$ & $274 \pm 71$ & $134 \pm 19$ & $81 \pm 13$ & $8.2 \pm 2.4$ & $250 \pm 40$ & $128 \pm 15$ & $79 \pm 11$ & $8.1 \pm 1.6$ & $258 \pm 42$ & $129 \pm 15$ & $78 \pm 12$ & $7.9 \pm 2.2$ & $255 \pm 41$ & $130 \pm 17$ & $77 \pm 11$ & $8.1 \pm 2.1$ \\
\hline $\begin{array}{c}p \text { vs } \\
\text { baseline }\end{array}$ & & & & & & $<.01^{* * *}$ & & $<.025^{* *}$ & & $<.025^{* *}$ & & & & $<.025^{* *}$ & & \\
\hline $\begin{array}{c}\text { SBP } \\
>130\end{array}$ & $233 \pm 86$ & $146 \pm 12$ & $84 \pm 13$ & $8.3 \pm 2.4$ & $202 \pm 43$ & $133 \pm 12$ & $79 \pm 10$ & $8.3 \pm 2.4$ & $208 \pm 28$ & $135 \pm 13$ & $78 \pm 13$ & $7.9 \pm 2.1$ & $205 \pm 45$ & $133 \pm 17$ & $76 \pm 11$ & $8.4 \pm 2.1$ \\
\hline $\begin{array}{c}p \text { vs } \\
\text { baseline }\end{array}$ & & & & & .5 & $<.0001^{* * * *}$ & $<.0001^{* * * *}$ & .06 & & $<.0001^{* * * *}$ & $<.01^{* * *}$ & $<.05^{*}$ & & $<.0001^{* * * *}$ & $<.01^{* * *}$ & \\
\hline $\begin{array}{l}\text { DBP } \\
>90\end{array}$ & $258 \pm 113$ & $155 \pm 18$ & $99 \pm 7$ & $8.4 \pm 2.6$ & $215 \pm 53$ & $140 \pm 14$ & $88 \pm 7$ & $7.5 \pm 1.8$ & $219 \pm 57$ & $137 \pm 15$ & $83 \pm 11$ & $7.9 \pm 2.2$ & $213 \pm 45$ & $139 \pm 14$ & $82 \pm 6$ & $9.2 \pm 2.7$ \\
\hline $\begin{array}{c}p \text { vs } \\
\text { baseline }\end{array}$ & & & & & & $<.01^{* * *}$ & $<.0001^{* * * *}$ & & & $<.01^{* * *}$ & $<.01^{* * *}$ & & & .06 & $<.01^{* * *}$ & \\
\hline $\begin{array}{c}\text { A1c } \\
>7.0\end{array}$ & $218 \pm 77$ & $134 \pm 19$ & $78 \pm 13$ & $9.6 \pm 2.0$ & $197 \pm 39$ & $131 \pm 15$ & $77 \pm 11$ & $9.0 \pm 1.9$ & $206 \pm 48$ & $132 \pm 13$ & $76 \pm 13$ & $9.3 \pm 2.1$ & $203 \pm 48$ & $131 \pm 13$ & $76 \pm 11$ & $9.3 \pm 1.7$ \\
\hline $\begin{array}{c}p \text { vs } \\
\text { baseline }\end{array}$ & & & & & & & & $<.01^{* * *}$ & & & & & & & & \\
\hline
\end{tabular}

Medication adherence was recorded at baseline. It is important to note that patients at baseline were asked only to complete the ARMS questionnaire without input from the Grand-Aides. Therefore, the baseline responses of 89 percent substantial adherence could not be verified by the Grand-Aides as they had not observed the adherence and this number could be a great deal lower. The Grand-Aides were able to verify adherence at 3 months of 98 percent, 6 months 97 percent, and 12 months 94 percent adherence.

Age, gender and race were not statistically different across outcomes from baseline and 1 year except: 1) Younger patients ( $<$ median of 62 years) weighed significantly $(p<.003)$ more at baseline than older patients $(260 \pm 89$ vs $200 \pm 44$ pounds) and younger patients lost significantly more weight from baseline to 12 months ( $260 \pm 89$ to $232 \pm 58, p<$ .001). 2) African American systolic blood pressure was significantly higher at baseline than for Caucasians $(139 \pm 17$ vs $124 \pm 16 \mathrm{mmHg}, p<.01)$ and African American patients also had a greater decrease in systolic blood pressure from baseline to 12 months $(139 \pm 17$ to $232 \pm 58, p<.025)$.

\subsection{Emergency department visits and 30-day readmis- sions}

In the 12 months before Grand-Aides, 28/48 patients (58 percent) had at least one non-trauma ED visit compared with 4
$14 / 48$ ( 29 percent) in the year after Grand-Aides. This is a 50 percent reduction in ED visits $(p<.01)$.

In the 12 months before Grand-Aides $6 / 48$ patients had at least 1 readmission (12.5 percent) compared with 3 patients (6.3 percent readmission rate) in the year after Grand-Aides. This is a 50 percent reduction although this did not achieve statistical significance. Of the 6 with readmissions before Grand-Aides, who might be considered the highest risk for readmission, none of them had a readmission after GrandAides.

At the end of the program a survey was sent to each of the participants to further quantify the impact of the GrandAides program on the participants. A total of 14 responses were received; 8 patients reported having a better understanding of their weight goal through the implementation of Grand-Aides, while one respondent stated that she/he did not remember a discussion of a specific weight goal (although this is part of the Grand-Aide protocol). Patients also compared their understanding of their related health condition (diabetes, hypertension, and/or obesity) prior to the implementation and after completion of the program with 14 participants noting their knowledge related to their hypertension had improved and 10 respondents noting their knowledge related to diabetes had improved by the end of the program. Twelve participants felt they had a better un- 
derstanding of what foods to eat at the completion of the program and 9 had a better understanding of how to take their medications. When questioned about their satisfaction with Grand-Aides, 12 reported being "very satisfied" and 2 reported being "satisfied". When it came to recommending the Grand-Aides model to others on a scale of 1 (not at all) to 5 (absolutely), 11 of the 12 respondents selected 5 and would absolutely recommend a friend to Grand-Aides if they had diabetes, hypertension or obesity.

\section{Discussion}

We have shown that with the utilization of Grand-Aides in patients with type II diabetes the following results may be noted:

1) Had 92 percent medication adherence at one year. In other studies, medication adherence has been notoriously poor, ranging from 7 percent to 62 percent adherent. ${ }^{[10]}$ Overall, less than 50 percent of patients take medications correctly for chronic disease. ${ }^{[11]}$

2) Grossman and Messerli ${ }^{[12]}$ found that with optimum management of patients with diabetes 30 percent of patients achieve control of hypertension. In our patients, fully 100 percent of the patients with abnormal diastolic blood pressure at baseline had normal diastolic blood pressure at 1 year; 48 percent of our patients with abnormal systolic hypertension at baseline under optimal treatment had normal blood pressure at 1 year. These finding lends credence to the extremely high reported medication adherence with Grand-Aides in this study.

3 ) In the US, the number of ED visits in patients with diabetes is increasing over the past 6 years now with more than a quarter of all ED visits for those $>45$ years old due to DM and more than $1 / 3$ of those over age $65 .^{[13]}$ Using Grand-Aides, we showed a 50 percent reduction in ED visits over a one-year follow-up period.

4) Others using teams of caregivers have achieved a reduction in 30-day all-cause readmissions of 30 percent. ${ }^{[14]}$ In our diabetic patients with Grand-Aides, hospital readmissions were significantly reduced by 50 percent.

5) Despite the Grand-Aides outstanding effects on adherence, blood pressure, ED visits and readmissions, the Hgb A1c of these patients did not change over 1 year. The average weight of our patients decreased by 230 to 217 pounds but with the wide standard deviation, the decrease was not statistically significant. It seems likely that since weight and $\mathrm{Hgb} \mathrm{A1c}$ are related, the Grand-Aides were less effective in changing eating habits than medication adherence that led to better

Published by Sciedu Press control of blood pressure, as well as reduction in ED visits and 30-day all-cause readmissions. These findings have caused us to re-examine the approach to weight loss in these patients. We are now adding further intervention including: (1) Formalizing the use of the Grand-Aides food labels. We have sent patients home with printed rolls of red, yellow and green food labels about the size of a U.S. quarter. They are "worth" 200 calories per serving for red, 140 for yellow (the calories on an 8-ounce sugared soda) and $<140$ for green. Patients and families have very much enjoyed the labeling, but we only have asked them to do this once as a "shock factor" of "everything is red" but we plan to follow-up with labeling what is bought for the first month at home. Such food labeling with red-yellow-green has been effective in changing choices in vending machines. ${ }^{[15]}$ (2) Going shopping with the patients to teach "perimeter shopping" staying to the healthy food around the perimeter of the store and using the red-yellow-green system on a shopping list; (3) We have created a program called "Yes you can" (C) in which we make a list of the foods the patient can eat at their favorite restaurants. (4) Finally, we will permit each patient to choose a diet that is nationally available and shown statistically in controlled studies to be associated with long-term weight loss. (5) Future opportunities should include an evaluation of available food options in food pantries and other community resources as well, with the ongoing plan to assist patients on choosing the healthiest options from those resources.

\subsection{Individual grand-aides treatment or group preven- tion for diabetes?}

The current work demonstrated that individualized contact with Grand-Aides was highly effective in addressing medication adherence and blood pressure control. Grand-Aides interventions also reduced ED visits and 30-day all-cause readmissions each by 50 percent with no readmissions in the "high risk" group that had a 30-day readmission the year before Grand-Aides.

In terms of the diabetes prevention programs using groups, Kahn and Davidson conclude, "Both the clinical trials and the community-based prevention studies have not shown a beneficial effect on any diabetes-related clinical outcome. While the goal of diabetes prevention is extremely important, the absence of any persuasive evidence for the effectiveness of community programs calls into question whether the use of public funds or national prevention initiatives should be supported at this time." ${ }^{[16]}$

Anecdotally, we have attempted to apply Grand-Aides in a group setting to treat patients with diabetes with less than satisfactory results. It seems that given our outstanding individual results, we will continue with application of Grand-Aides 
to individual patients.

\subsection{Cost savings}

We did not measure specific expenses before or after the use of Grand-Aides. Better control of diabetic patients will result in more medication expense, but fewer clinic visits, ED visits and hospitalizations. In patients "dually eligible" for Medicare and Medicaid, successful treatment of hypertension was associated with an estimated $\$ 10$ billion reduction in expense for the U.S. ${ }^{[15]}$ Depending upon the parameters of treatment, such successful treatment may result in loss of income if payment is fee-for-service and readmissions and ED visits decrease. On the other hand, Grand-Aides will result in savings for a health plan, insurer or employer if they are "at risk" for the payment, meaning that payment is delivered as either capitated or bundled payment. In the recently published study from the University of Virginia in patients with heart failure, net savings were $\$ 562,000$ per Grand-Aide per year.

\subsection{Limitations}

This study was based on historical controls, albeit with the same patients. While this is not ideal, the other types of controls also have their issues: 1) A randomized clinical trial deprives half the people from an intervention that was shown to be highly effective in a similar population of patients; 2) As all patients who were identified to be "high risk" were asked to participate in the program, concurrent controls could only have been done in patients who refused a Grand-Aide, and potentially defines a group that differs significantly from those who accepted Grand-Aides.

This was the first application of Grand-Aides in which patients were enrolled in the clinic at a time distant from any hospitalization. Hospital discharge certainly focuses the at- tention of the patient and family on doing what they can to avoid readmission and may become more attentive to all aspects of their management (including diet and exercise) than those chosen as of a particular date in the clinic or from a registry. We will spend even more time working with such patients in the future to be certain they are attentive to all parts of their management regardless of the time of their enrollment and whether "they think they need" the intervention.

\section{Conclusions}

The Grand-Aides program with one-on-one, personalized home visits was effective in changing behavior in diabetic patients. This behavior change has been associated with results for medication adherence, blood pressure control, and reductions in ED visits and readmissions that compare favorably with published comparative data. For systems "at risk" for patient expense, the Grand-Aides program will result in significant savings.

\section{ACKNOWLEDGEMENTS}

Rebekah Compton was responsible for the manuscript and the data; she was Grand-Aides supervisor in the second part of the program. Kimberly Bednar was Grand-Aides supervisor in the beginning of the program; she provided significant input on the manuscript. Peggie E. Donowitz was the teacher and overseer of implementation of the program from Grand-Aides USA; she provided input on the manuscript. M. Norman Oliver brought the program to Family Medicine and UVA and modified as necessary; he provided input on the manuscript. Kawai Tanabe, MPH assisted with data analysis.

\section{CONFLICTS OF INTEREST DisClOSURE}

The authors declare that there is no conflict of interest.

\section{REFERENCES}

[1] O’Neil PM, Miller-Kovach K, Tuerk PW, et al. Randomized controlled trial of a nationally available weight control program tailored for adults with type 2 diabetes. Obesity [Internet]. 2016 Nov [cited 2018 Dec 28]; 24(11): 2269-77. PMid:27804264 https: //doi.org/10.1002/oby. 21616

[2] Polonsky WH, Henry RR. Poor medication adherence in type 2 diabetes: recognizing the scope of the problem and its key contributors. Patient Prefer Adherence. 2016; 10: 1299-307. PMid:27524885 https://doi.org/10.2147/PPA.S106821

[3] Ye Q, Khan U, Boren SA, et al. An Analysis of Diabetes Mobile Applications Features Compared to AADE7TM: Addressing Self-Management Behaviors in People With Diabetes. J Diabetes Sci Technol. 2018 Jul; 12(4): 808-16. PMid:29390917 https: //doi.org/10.1177/1932296818754907
[4] Rubin DJ. Correction to: Hospital Readmission of Patients with Diabetes. Curr Diab Rep. 2018 Apr; 18(4): 21. PMid:29536197 https : //doi.org/10.1007/s11892-018-0989-1

[5] American Diabetes Association. Standards of Medical Care in Diabetes. 2017.

[6] Thomas SC, Greevy RA, Garson A. Effect of Grand-Aides Nurse Extenders on Readmissions and Emergency Department Visits in Medicare Patients With Heart Failure. Am J Cardiol [Internet]. 2018 Jun [cited 2018 Oct 9]; 121(11): 1336-42. PMid:29627108 https ://doi.org/10.1016/j.amjcard.2018.02.012

[7] Phillips RA. America's healthcare transformation: strategies and innovations.

[8] Grand-Aides USA. Grand-Aides program.

[9] Kripalani S, Risser J, Gatti ME, et al. Development and Evaluation of the Adherence to Refills and Medications Scale (ARMS) among Low- 
Literacy Patients with Chronic Disease. Value Heal. 2009 Jan; 12(1): 118-23. PMid:19911444 https://doi .org/10.1111/j .1524-4 733.2008.00400.x

[10] Dudley-Brown S. The Importance of Medication Adherence.

[11] Vinogradova Y, Coupland C, Brindle P, Hippisley-Cox J. Patients who discontinued statin treatment: a protocol for cohort study using primary care data. BMJ Open. 2015 Oct; 5(10): e008701. PMid:26493458 https://doi.org/10.1136/bmjopen-2015-0 08701

[12] Grossman A, Grossman E. Blood pressure control in type 2 diabetic patients. Cardiovasc Diabetol [Internet]. 2017 [cited 2018 Dec 28]; 16(1): 3. PMid:28056987 https://doi.org/10.1186/s12933 $-016-0485-3$

[13] Emergency Room Visits Jump Among Diabetics [Internet]. [cited 2018 Dec 28]. Available from: https: //www . aarp.org/health/conditions-treatments/in fo-2018/diabetics-er-visits.html
[14] Bansal V, Mottalib A, Pawar TK, et al. Inpatient diabetes management by specialized diabetes team versus primary service team in non-critical care units: impact on 30-day readmission rate and hospital cost. BMJ Open Diabetes Res Care [Internet]. 2018 Apr 5 [cited 2018 Dec 28]; 6(1): e000460. PMid:29657719 https: //doi.org/10.1136/bmjdrc-2017-000460

[15] Reducing Obesity: Policy Strategies from the Tobacco Wars I Urban Institute [Internet]. [cited 2018 Dec 28]. Available from: https://www.urban.org/research/publication/red ucing-obesity-policy-strategies-tobacco-wars

[16] Kahn R, Davidson MB. The Reality of Type 2 Diabetes Prevention Diabetes Care [Internet]. 2014 Apr 20 [cited 2018 Dec 28]; 37(4): 943-9. PMid:24652724 https ://doi .org/10.2337/dc13-1954

[17] Dickerson, et al. Glucose control and vascular complications in veterans with type 2 diabetes. New England Journal of Medicine. 2009 Jan 8; 360(2): 129-139. 